Pacific

Journal of

Mathematics

GROMOV HYPERBOLIC GROUPS AND THE MACAEV NORM RUI OKAYASU

Volume $223 \quad$ No. 1

January 2006 


\title{
GROMOV HYPERBOLIC GROUPS AND THE MACAEV NORM
}

\author{
RUI OKAYASU
}

\begin{abstract}
Let $\Gamma$ be a Gromov hyperbolic group with a finite set $A$ of generators. We prove that $h_{\text {top }}(\Sigma(\infty)) \leq k_{\infty}^{-}\left(\lambda_{A}\right) \leq \operatorname{gr}(\Gamma, A)$, where $\operatorname{gr}(\Gamma, A)$ is the growth entropy, $\boldsymbol{h}_{\text {top }}(\Sigma(\infty))$ is the Coornaert-Papadopoulos topological entropy of the subshift $\Sigma(\infty)$ associated with $(\Gamma, A)$, and $k_{\infty}^{-}\left(\lambda_{A}\right)$ is Voiculescu's numerical invariant, which is an obstruction to the existence of quasicentral approximate units relative to the Macaev norm for a tuple of unitary operators $\lambda_{A}=\left(\lambda_{a}\right)_{a \in A}$ in the left regular representation of $\Gamma$. We also prove that these three quantities are equal for a hyperbolic group splitting over a finite group.
\end{abstract}

\section{Introduction}

Let $\Gamma$ be a finitely generated group with a finite generating set $A$. We consider the family $\lambda_{A}=\left(\lambda_{a}\right)_{a \in A}$ of left translation operators on $\ell^{2}(\Gamma)$, specifically the value of Voiculescu's numerical invariant $k_{\infty}^{-}$for this family. Voiculescu introduced this invariant $k_{\infty}^{-}(\tau)$, for a tuple $\tau$ of Hilbert space operators, in a remarkable series of papers $[1979 ; 1981 ; 1990$; David and Voiculescu 1990] to deal with perturbation problems.

For the case of free groups, Voiculescu gave an estimate for $k_{\infty}^{-}\left(\lambda_{A}\right)$; we obtain its exact value. For the case of certain amalgamated free product groups, we proved in [Okayasu 2004] that $k_{\infty}^{-}\left(\lambda_{A}\right)$ equals the growth entropy $\operatorname{gr}(\Gamma, A)$ of $\Gamma$ with respect to $A$. These groups are Gromov hyperbolic groups in the sense of [Gromov 1987]. In [Okayasu 2004], we showed that if a subshift $\Sigma$ satisfies a certain condition, then $k_{\infty}^{-}(\tau)=h_{\text {top }}(\Sigma)$ for the family $\tau$ of creation operators on the Fock space associated with $\Sigma$, which is used to define the Matsumoto algebra [1997] associated to $\Sigma$. (Here $h_{\text {top }}(\Sigma)$ is the topological entropy of $\Sigma$.) This equation holds for every shift of finite type.

M. Coornaert and A. Papadopoulos [2001] have shown the following: Let $X$ be a proper geodesic metric space that is $\delta$-hyperbolic. The class of functions on $X$ called horofunctions (a generalization of Busemann functions) gives a description of the boundary at infinity $\partial X$. When $X$ is the Cayley graph of a hyperbolic group

MSC2000: primary 47B10; secondary 47A30, 37B10, $20 \mathrm{~F} 65$.

Keywords: perturbation theory, Macaev ideal, hyperbolic groups. 
$\Gamma$, the space of cocycles associated with horofunctions that take integral values on the vertices is a shift of finite type $\Sigma(\infty)$. (See also [Gromov 1987].)

Continuing this line of investigation, we first determine(Theorem 1.1) a lower bound for $k_{\infty}^{-}\left(\lambda_{A}\right)$ in terms of the topological entropy $h_{\text {top }}(\Sigma(\infty))$, for arbitrary hyperbolic groups. We therefore have

$$
h_{\text {top }}(\Sigma(\infty)) \leq k_{\infty}^{-}\left(\lambda_{A}\right) \leq \operatorname{gr}(\Gamma, A),
$$

since the upper bound was already given in [Okayasu 2004]. We also show here that if a given hyperbolic group $\Gamma$ splits over a finite group, the equation $h_{\text {top }}(\Sigma(\infty))=$ $\operatorname{gr}(\Gamma, A)$ holds for a certain finite generating set $A$ of $\Gamma$ (Corollary 1.2). As a consequence, the inequalities turn into an equalities for such groups:

$$
h_{\text {top }}(\Sigma(\infty))=k_{\infty}^{-}\left(\lambda_{A}\right)=\operatorname{gr}(\Gamma, A) .
$$

It was already known from [Voiculescu 1993] that $k_{\infty}^{-}\left(\lambda_{A}\right) \neq 0$ for every nonelementary hyperbolic group $\Gamma$, because $\Gamma$ is nonamenable.

Notation. We denote by $\Sigma(\infty)$ the shift of finite type relative to $(\Gamma, A)$, constructed in [Coornaert and Papadopoulos 2001].

Theorem 1.1. Let $\Gamma$ is a Gromov hyperbolic group with a finite generating set $A$ and $\lambda$ its left regular representation. Set $\lambda_{A}=\left(\lambda_{a}\right)_{a \in A}$. Then we have

$$
h_{\text {top }}(\Sigma(\infty)) \leq k_{\infty}^{-}\left(\lambda_{A}\right) \leq \operatorname{gr}(\Gamma, A) .
$$

Corollary 1.2. Let $\Gamma$ is a nonelementary hyperbolic group with a finite generating set $A, \lambda$ its left regular representation and $\lambda_{A}=\left(\lambda_{a}\right)_{a \in A}$. Suppose that either

(1) $\Gamma$ can be written nontrivially as a free product $G_{1} * G_{2}$ and $A=F_{1} \cup F_{2}$ for some finite generating sets $F_{1}, F_{2}$ of $G_{1}, G_{2}$; or

(2) $\Gamma$ has a form of a free product $G_{1} *_{H} G_{2}$ with finite amalgamated subgroup $H$, which is properly contained in both factors and of index greater than 2 in at least one factor, and $A=F_{1} \cup F_{2}$ for some finite generating sets $F_{1}, F_{2}$ of $G_{1}, G_{2}$, containing $H$; or

(3) $\Gamma$ is an HNN extension

$$
\left.G *_{H} \theta=\langle G, x| h x=x \theta(h) \text { for } h \in H\right\rangle,
$$

where $H$ is a proper finite subgroup of $G$ and $A=F \cup\left\{x, x^{-1}\right\}$ for some finite generating set $F$ of $G$, which contains both $H$ and $\theta(H)$.

Then $k_{\infty}^{-}\left(\lambda_{A}\right)=\operatorname{gr}(\Gamma, A)=h_{\text {top }}(\Sigma(\infty))$. 


\section{Preliminaries}

Voiculescu's perturbation theory. Let $\mathscr{H}$ be a separable infinite dimensional Hilbert space and let $\mathbb{B}(\mathscr{H}), \mathbb{K}(\mathscr{H})$ denote, respectively, the spaces of bounded linear operators and compact operators on $\mathscr{H}$. A symmetrically normed ideal $\left(\mathfrak{S},\|\cdot\|_{\mathfrak{S}}\right)$ is an ideal $\mathfrak{S}$ of $\mathbb{K}(\mathscr{H})$ which is a Banach space endowed with the norm $\|\cdot\|_{\mathfrak{S}}$ satisfying

$$
\|X T Y\|_{\mathfrak{S}} \leq\|X\| \cdot\|T\|_{\mathfrak{S}} \cdot\|Y\|
$$

for $T \in \mathfrak{S}$ and $X, Y \in \mathbb{B}(\mathscr{H})$, where $\|\cdot\|$ is the operator norm on $\mathbb{B}(\mathscr{H})$.

It is well-known that the Schatten $p$-classes $\mathscr{C}_{p}(\mathscr{H})$ are symmetrically normed ideals. So are the ideals $\mathscr{C}_{p}^{-}(\mathscr{H})$ defined for $1 \leq p \leq \infty$ by the norm

$$
\|T\|_{p}^{-}=\sum_{j=1}^{\infty} \lambda_{j} j^{-1+1 / p}
$$

(where $\lambda_{1} \geq \lambda_{2} \geq \cdots$ are the eigenvalues of $\left(T^{*} T\right)^{1 / 2}$ ); they are important for perturbation theory. The particular case $\mathscr{C}_{\infty}^{-}(\mathscr{H})$ is also known as the Macaev ideal. Note that $\mathscr{b}_{1}^{-}(\mathscr{H})=\mathscr{C}_{1}(\mathscr{H})$ but

$$
\mathscr{C}_{p}^{-}(\mathscr{H}) \varsubsetneqq \mathscr{C}_{p}(\mathscr{H}) \varsubsetneqq \mathscr{C}_{q}^{-}(\mathscr{H}) \quad \text { if } 1<p<q .
$$

The dual $\mathfrak{S}^{*}$, where the duality is given by the bilinear form $(X, Y) \mapsto \operatorname{Tr}(X Y)$, is again a normed ideal. We have $\mathscr{C}_{p}(\mathscr{H})^{*}=\mathscr{C}_{q}(\mathscr{H})$, where $p>1$ and $1 / p+1 / q=1$. Moreover $\mathscr{C}_{p}^{-}(\mathscr{H})^{*}=\mathscr{C}_{q}^{+}(\mathscr{H})$, where $\mathscr{C}_{q}^{+}(\mathscr{H})$ consists of all $T \in \mathbb{K}(\mathscr{H})$ such that

$$
\|T\|_{q}^{+}=\sup _{k} \frac{\sum_{j=1}^{k} \lambda_{j}}{\sum_{j=1}^{k} j^{-1 / q}}<\infty .
$$

Let $\mathfrak{S}$ be a symmetrically normed ideal of $\mathbb{K}(\mathscr{H})$. For an $N$-tuple $\tau=\left(T_{1}, \ldots, T_{N}\right)$ of bounded linear operators on $\mathscr{H}$, we define

$$
k_{\mathfrak{S}}(\tau)=\liminf _{A \in \mathbb{F}(H)_{1}^{+}} \max _{1 \leq i \leq N}\left\|\left[A, T_{i}\right]\right\|_{\mathfrak{S}},
$$

where the inferior limit is taken with respect to the natural order on

$$
\mathbb{F}(H)_{1}^{+}=\{T \in \mathbb{K}(\mathscr{H}) \mid T: \text { finite rank, } 0 \leq T \leq I\}
$$

and $[A, B]=A B-B A$. We write $k_{p}^{-}(\tau)$ when $\mathfrak{S}=\mathscr{C}_{p}^{-}(\mathscr{H})$.

We see from the definition that $k_{\mathfrak{S}}(\tau)$ measures the obstruction to the existence of a sequence $\left\{A_{n}\right\}_{n=1}^{\infty} \subseteq \mathbb{F}(\mathscr{H})_{1}^{+}$such that $A_{n} \nearrow I$ and $\lim _{n \rightarrow \infty}\left\|\left[A_{n}, T_{i}\right]\right\|_{\Phi}=0$ for $1 \leq i \leq N$. If such a sequence exists, it is called a quasicentral approximate unit for $\tau$ relative to $\mathfrak{S}$. 
Proposition 2.1 [Voiculescu 1990, Proposition 2.1]. Let $\tau=\left(T_{1}, \ldots, T_{N}\right) \in \mathbb{B}(\mathscr{H})^{N}$ and $X_{i} \in \mathscr{C}_{1}^{+}(\mathscr{H})$ for $i=1, \ldots, N$. If

$$
\sum_{i=1}^{N}\left[X_{i}, T_{i}\right] \in \mathscr{C}_{1}(\mathscr{H})+\mathbb{B}(\mathscr{H})_{+},
$$

then

$$
\left|\operatorname{Tr}\left(\sum_{i=1}^{N}\left[X_{i}, T_{i}\right]\right)\right| \leq k_{\infty}^{-}(\tau) \sum_{a=1}^{N}\left\|X_{i}\right\|_{1}^{\tilde{+}},
$$

where $\left\|X_{i}\right\|_{1}^{\tilde{+}}=\inf _{Y \in \mathbb{E}(\mathscr{H})}\left\|X_{i}-Y\right\|_{1}^{+}$.

Proposition 2.2 [Gohberg and Krĕn 1969, Theorem 14.1]. For $T \in \mathscr{C}_{1}^{+}(\mathscr{H})$, we have

$$
\|T\|_{1}^{\widetilde{+}}=\limsup _{n \rightarrow \infty} \frac{\sum_{j=1}^{n} s_{j}(T)}{\sum_{j=1}^{n} 1 / j} .
$$

Subshifts. We briefly define the necessary concepts from symbolic dynamics; see [Lind and Marcus 1995] for a more leisurely introduction.

Let $\mathscr{A}$ be a finite alphabet and $\mathscr{A}^{\mathbb{N}}$ the one-sided infinite product space $\prod_{i=0}^{\infty} \mathscr{A}$ with the product topology (of discrete topologies). The shift map $\sigma$ on $\mathscr{A}^{\mathbb{N}}$ is given by $(\sigma(x))_{i}=x_{i+1}$ for $i \in \mathbb{N}$. A word over $\mathscr{A}$ is a finite sequence $w=\left(a_{1}, \ldots, a_{n}\right)$ with $a_{i} \in \mathscr{A}$. For $x \in \mathscr{A}^{\mathbb{N}}$ and a word $w=\left(a_{1}, \ldots, a_{n}\right)$, we say that $w$ occurs in $x$ if there is an index $i$ such that $x_{i}=a_{1}, \ldots, x_{i+n-1}=a_{n}$. For a collection $\mathscr{F}$ of words over $\mathscr{A}^{\mathbb{N}}$, we define the (one-sided) subshift $X=X_{\mathscr{F}}$ to be the subset of sequences in $\mathscr{A}^{\mathbb{N}}$ in which no word in $\mathscr{F}$ occurs.

Let $X$ be a subshift of $\mathscr{A}^{\mathbb{N}}$. We denote by $\mathcal{W}_{n}(X)$ the set of all words with length $n$ that occur in $X$ and we set

$$
\mathcal{W}(X)=\bigcup_{n=0}^{\infty} \mathcal{W}_{n}(X) .
$$

Let $\varphi: \mathscr{W}_{m+n+1}(X) \rightarrow \mathscr{A}$ be a map, which we call a block map. The extension of $\varphi$ from $X$ to $\mathscr{A}^{\mathbb{N}}$ is defined by $\left(x_{i}\right)_{i \in \mathbb{N}} \mapsto\left(y_{i}\right)_{i \in \mathbb{N}}$, where

$$
y_{i}=\varphi\left(\left(x_{i-m}, x_{i-m+1}, \ldots, x_{i+n}\right)\right) .
$$

We also denote this extension by $\varphi$ and call it a sliding block code.

The topological entropy of a subshift $X$ is defined by

$$
h_{\text {top }}(X)=\lim _{n \rightarrow \infty} \frac{1}{n} \log \operatorname{card} W_{n}(X) .
$$

A simple class of subshifts is that of shifts of finite type (SFT), those that can be described by a finite set of forbidden words. Let $M=[M(a, b)]_{a, b \in \mathscr{A}}$ be a $0-1$ 
matrix. Then

$$
\Sigma_{M}:=\left\{\left(x_{i}\right)_{i=0}^{\infty} \in \mathscr{A}^{\mathbb{N}} \mid M\left(x_{i}, x_{i+1}\right)=1\right\}
$$

is called the one-sided topological Markov shift by $M$ and it is a shift of finite type.

Gromov hyperbolic groups. For basic facts about Gromov hyperbolic spaces and groups, see [Gromov 1987] and [Coornaert and Papadopoulos 1993].

Let $(X,||)$ be a metric space which is proper, geodesic and $\delta$-hyperbolic for some $\delta \geq 0$. A function $f: X \rightarrow \mathbb{R}$ is $\varepsilon$-convex, where $\varepsilon \geq 0$, if for any geodesic segment $\left[x_{0}, x_{1}\right]$ in $X$ and any $t \in[0,1]$, we have

$$
f\left(x_{t}\right) \leq(1-t) f\left(x_{0}\right)+t f\left(x_{1}\right)+\varepsilon,
$$

where $x_{t}$ is the point on $\left[x_{0}, x_{1}\right]$ satisfying $\left|x_{0}-x_{t}\right|=t\left|x_{0}-x_{1}\right|$.

Definition 2.3. Let $\varepsilon \geq 0$. An $\varepsilon$-horofunction on $X$ is an $\varepsilon$-convex function $h: X \rightarrow \mathbb{R}$ satisfying $h(x)-\lambda=\operatorname{dist}\left(x, h^{-1}(\lambda)\right)$ for every $x \in X$ and $\lambda \in \mathbb{R}$ such that $h(x) \geq \lambda$.

Definition 2.4. Let $r:[0, \infty) \rightarrow X$ be a geodesic ray. The associated Busemann function $h_{r}: X \rightarrow \mathbb{R}$ is defined by

$$
h_{r}(x)=\lim _{t \rightarrow \infty}|x-r(t)|-t .
$$

A Busemann function on a $\delta$-hyperbolic $X$ is a $4 \delta$-horofunction [Coornaert and Papadopoulos 2001, Proposition 2.5]. Thus Busemann functions form an important class of horofunctions.

Definition 2.5. A function $\varphi: X \times X \rightarrow \mathbb{R}$ is called an $\varepsilon$-cocycle if there is an $\varepsilon$-horofunction $h: X \rightarrow \mathbb{R}$ such that

$$
\varphi(x, y)=h(x)-h(y)
$$

for every $x, y \in X$. We call such a function $h$ a primitive for $\varphi$. (If $h$ is a primitive for $\varphi$, so is $h+c$ for any constant $c$.)

Proposition 2.6 [Coornaert and Papadopoulos 2001, Proposition 2.7]. Let $\varphi$ be a cocycle on $X$. For $x, y, z$ and $w \in X$, we have

(1) $\varphi(x, x)=0$,

(2) $\varphi(x, y)=-\varphi(y, x)$,

(3) $\varphi(x, y)=\varphi(x, z)+\varphi(z, y)$,

(4) $|\varphi(x, y)| \leq|x-y|$,

(5) $|\varphi(x, y)-\varphi(z, w)| \leq|x-z|+|y-w|$. 
Let $\gamma$ be an isometry of $X, h: X \rightarrow \mathbb{R}$ an $\varepsilon$-horofunction and $\varphi: X \times X \rightarrow \mathbb{R}$ an $\varepsilon$-cocycle. The functions $\gamma h$ and $\gamma \varphi$ defined by

$$
\gamma h(x)=h\left(\gamma^{-1} x\right), \quad \gamma \varphi(x, y)=\varphi\left(\gamma^{-1} x, \gamma^{-1} y\right),
$$

for $x, y \in X$, are an $\varepsilon$-horofunction and an $\varepsilon$-cocycle, respectively. If $\varphi$ is the cocycle of $h$, then $\gamma \varphi$ is the cocycle of $\gamma h$. Let $\Phi$ be the set of $\varepsilon$-cocycles on $X$ for all possible values of $\varepsilon \geq 0$. We equip $\Phi$ with the topology of uniform convergence on compact sets.

Definition 2.7. Let $\varphi$ be a cocycle on $X$. A $\varphi$-gradient arc is a path $g: I \rightarrow X$, parameterized by arclength, satisfying

$$
\varphi\left(g(t), g\left(t^{\prime}\right)\right)=t^{\prime}-t
$$

for every $t, t^{\prime} \in I$. If $I=\mathbb{R}$ or $I=[0, \infty)$, we say that $g$ is a $\varphi$-gradient line or ray, respectively. If $g(0)=x$, we say that $g$ starts at $x$.

Lemma 2.8 [Coornaert and Papadopoulos 2001, Lemma 2.9]. Let $\varphi$ be a cocycle on $X$ and $I \subseteq \mathbb{R}$ an interval with $a \in I, I_{1}=I \cap(-\infty, a]$ and $I_{2}=I \cap[a, \infty)$. If $g: I \rightarrow X$ is a path whose restrictions to $I_{1}$ and $I_{2}$ are $\varphi$-gradient arcs, then $g$ is itself a $\varphi$-gradient arc.

Proposition 2.9 [Coornaert and Papadopoulos 2001, Proposition 2.10]. Let $\varphi$ be a cocycle on $X$.

(1) Any $\varphi$-gradient arc $g: I \rightarrow X$ is a geodesic.

(2) If $x, y \in X$ satisfying $\varphi(x, y)=|x-y|$, and if $g:[a, b] \rightarrow X$ is a geodesic joining $x$ and $y$, then $g$ is a $\varphi$-gradient arc.

Proposition 2.10 [Coornaert and Papadopoulos 2001, Proposition 2.13]. For every cocycle $\varphi$ on $X$ and for every $x \in X$, there is a $\varphi$-gradient ray $g:[0, \infty) \rightarrow X$ starting at $x$.

Let $\varphi$ be a cocycle on $X$ and $g:[0, \infty) \rightarrow X$ a $\varphi$-gradient ray. By Proposition 2.9, part (1), $g$ is a geodesic and so converges to a well-defined point $g(\infty) \in \partial X$.

Proposition 2.11 [Coornaert and Papadopoulos 2001, Proposition 3.1]. Let $\varphi$ be a cocycle on $X$ and let $g, g^{\prime}:[0, \infty) \rightarrow X$ be $\varphi$-gradient rays. Then $g(\infty)=g^{\prime}(\infty)$.

Definition 2.12. We define a map $\pi: \Phi \rightarrow \partial X$ by setting $\Phi(\varphi)=g(\infty) \in \partial X$, where $g:[0, \infty) \rightarrow X$ is a $\varphi$-gradient ray.

Let $\operatorname{Isom}(X)$ denote the group of isometries of $X$. The action of $\operatorname{Isom}(X)$ on $\Phi$ defined by $(\gamma, \varphi) \mapsto \gamma \varphi$ is continuous.

Proposition 2.13 [Coornaert and Papadopoulos 2001, Proposition 3.3]. The map $\pi: \Phi \rightarrow \partial X$ is continuous, surjective, and commutes with the actions of $\operatorname{Isom}(X)$ on $\Phi$ and $\partial X$. 
For any cocycle $\varphi$, any geodesic ray $r:[0, \infty) \rightarrow X$ satisfying $r(\infty)=\pi(\varphi)$, and any $t \geq 0$, we set

$$
R_{\varphi, t}=\{x \in X \mid \varphi(x, r(t))=0\} \cap B(r(t), 16 \delta) .
$$

Proposition 2.14 [Coornaert and Papadopoulos 2001, Proposition 3.4]. For $\varphi \in \Phi$, let $r:[0, \infty) \rightarrow X$ be a geodesic ray such that $r(\infty)=\pi(\varphi)$. For all $x \in X$ and $t \in \mathbb{R}$ satisfying $t>|x-r(0)|+16 \delta$, we have

$$
\varphi(x, r(t))=\operatorname{dist}\left(x, R_{\varphi, t}\right) .
$$

In all that follows, $\Gamma$ is a $\delta$-hyperbolic group with respect to a finite set of generators $A$ and $X$ is the Cayley graph associated to the pair $(\Gamma, A)$. We denote by $X^{0}=\Gamma$ the set of vertices and by $X^{1}$ the set of edges of $X$. For $x \in \Gamma$, we denote by $|x|$ the word length of $x$ with respect to $A$.

Definition 2.15. A horofunction $h: X \rightarrow \mathbb{R}$ is said to be integral if $h(x) \in \mathbb{Z}$ for every $x \in X^{0}$. A cocycle having an integral horofunction as a primitive is called an integral cocycle.

Every integral cocycle is completely determined by its values on $\Gamma \times \Gamma$, by [Coornaert and Papadopoulos 2001, Corollary 4.4]. Thus we can regard an integral cocycle on $X$ as a function from $\Gamma \times \Gamma$ to $\mathbb{Z}$. Let $\Phi_{0} \subseteq \Phi$ be the space of integral cocycles on $X$. The topology induced on $\Phi_{0}$ by $\Phi$ is the topology of pointwise convergence on $\Gamma \times \Gamma$. For simplicity, we denote by $\pi: \Phi_{0} \rightarrow \partial \Gamma$ the restriction of the map $\pi: \Phi \rightarrow \partial \Gamma$.

Proposition 2.16 [Coornaert and Papadopoulos 2001, Proposition 4.5]. The map $\pi: \Phi_{0} \rightarrow \partial \Gamma$ is continuous, $\Gamma$-equivalent, surjective and uniformly finite to one. In fact, for every $\xi \in \partial \Gamma$ we have

$$
\operatorname{card}\left\{\varphi \in \Phi_{0} \mid \pi(\varphi)=\xi\right\} \leq\left(2 N_{0}+1\right)^{N_{1}},
$$

where $N_{0}$ is the integral part of $16 \delta+1$ and $N_{1}$ is the number of elements in $\Gamma$ contained in the closed ball of radius $N_{0}$ centered at the identity.

Lemma 2.17 [Coornaert and Papadopoulos 2001, Lemma 5.1]. For every $\varphi \in \Phi_{0}$ and $x \in X^{0}$, there is $a \in A$ such that $\varphi(x, x a)=1$.

Now we fix a total order relation on the finite generating set $A$. Let $\varphi \in \Phi_{0}$ and $x \in X^{0}$. The lexicographic order on $A^{\mathbb{N}}$ induces a total order on the set of $\varphi$-gradient rays starting at $x$.

Proposition 2.18 [Coornaert and Papadopoulos 2001, Proposition 5.2]. Let $\varphi \in \Phi_{0}$ and $x \in X^{0}$. The set of $\varphi$-gradient rays starting at $x$ has a smallest element.

Definition 2.19. We define a map $\alpha: \Phi_{0} \rightarrow \Phi_{0}$ by $\alpha(\varphi)=a^{-1} \varphi$, where $\varphi \in \Phi_{0}$ and $a$ is the smallest element in $A$ satisfying $\varphi(e, a)=1$. 
Proposition 2.20 [Coornaert and Papadopoulos 2001, Proposition 5.6]. The map $\alpha: \Phi_{0} \rightarrow \Phi_{0}$ is continuous.

Proposition 2.21 [Coornaert and Papadopoulos 2001, Proposition 5.7]. Let $\varphi \in \Phi_{0}$ and $g:[0, \infty) \rightarrow X$ be the smallest $\varphi$-gradient ray starting at e. For $n \in \mathbb{N}$, let $a_{n} \in A$ be the label of the oriented edge from $g(n)$ to $g(n+1)$ and $g_{n}:[0, \infty) \rightarrow X$ the smallest $\alpha^{n}(\varphi)$-gradient ray starting at $e$.

(1) $\alpha^{n}(\varphi)=g(n)^{-1} \varphi$.

(2) $g_{n}(t)=g(n)^{-1} g(t+n)$ for any $t \in[0, \infty)$.

(3) For every $k \in \mathbb{N}$, the label of oriented edge from $g_{n}(k)$ to $g_{n}(k+1)$ is $a_{k+n}$.

Next we introduce the shift of finite type $(\Sigma(\infty), T)$ and the conjugacy $P$ from $\left(\Phi_{0}, \alpha\right)$ to $(\Sigma(\infty), T)$. We take integers $R \geq 100 \delta+1$ and $L \geq 2 R+32 \delta+1$. For a subset $Y \subseteq X$ and $\varepsilon \geq 0$, we set

$$
N(Y, \varepsilon)=\{x \in X \mid \operatorname{dist}(x, Y) \leq \varepsilon\} .
$$

For $\varphi \in \Phi_{0}$, let $g:[0, \infty) \rightarrow X$ be the smallest $\varphi$-gradient ray starting at $e$. Set

$$
V(\varphi)=N(g([0, L]), R) .
$$

$V(\varphi)$ is contained in the closed ball $B(e, L+R)$ of radius $L+R$ centered at $e$.

For each $\varphi \in \Phi_{0}$, we define a function $\rho(\varphi): V(\varphi) \rightarrow \mathbb{R}$ by

$$
\rho(\varphi)(x)=\varphi(x, e)
$$

for $x \in V(\varphi)$. Note that $\rho(\varphi)$ is the restriction to $V(\varphi)$ of the primitive $h$ of $\varphi$ with $h(e)=0$. We set

$$
S=\left\{\rho(\varphi): V(\varphi) \rightarrow \mathbb{R} \mid \varphi \in \Phi_{0}\right\} .
$$

Lemma 2.22 [Coornaert and Papadopoulos 2001, Lemma 6.2]. The set $S$ is finite.

Definition 2.23. Let $\Sigma$ be the set of sequences $\left(\sigma_{n}\right)_{n \geq 0}$ with $\sigma_{n} \in S$ for $n \geq 0$, and give it the product topology (of discrete topologies on copies of $S$ ). The map $T: \Sigma \rightarrow \Sigma$ is the shift map. Define a map $P: \Phi_{0} \rightarrow \Sigma$ by

$$
\Phi_{0} \ni \varphi \mapsto\left(\sigma_{n}\right)_{n \geq 0} \in \Sigma,
$$

where $\sigma_{n}=\rho\left(\alpha^{n}(\varphi)\right)$ for $n \geq 0$.

Let $s \in S$. We denote by $V(s)$ the domain of the function $s$. Since $R \geq 1$, the domain $V(s)$ contains the closed unit ball $B(e, 1)$. Hence the value $s(a)$ is welldefined for all $a \in A$. Since the finite generating set $A$ is equipped with a fixed total order relation, we can define $w(s)$ to be the smallest element $a \in A$ satisfying $s(a)=-1$. (Such an $a$ exists because of Lemma 2.17.) 
Let $\sigma=\left(\sigma_{n}\right)_{n \geq 0} \in \Sigma$. We define a sequence $\left(\gamma_{n}(\sigma)\right)_{n \geq 0}$ by setting

$$
\gamma_{0}(\sigma)=e, \quad \gamma_{n}(\sigma)=w\left(\sigma_{0}\right) \cdots w\left(\sigma_{n-1}\right) \quad \text { for } n \geq 1 .
$$

For $n \geq 0$, we set

$$
V_{n}(\sigma)=\gamma_{n}(\sigma) V\left(\sigma_{n}\right)
$$

This depends only on the first $n+1$ coordinates of $\sigma$. We also define functions $f_{n}(\sigma): V_{n}(\sigma) \rightarrow \mathbb{R}$ by $f_{n}(\sigma)(x)=\sigma_{n}\left(\gamma_{n}(\sigma)^{-1} x\right)-n$ for $x \in V_{n}(\sigma)$.

Lemma 2.24 [Coornaert and Papadopoulos 2001, Lemma 6.5]. For $\varphi \in \Phi_{0}$, take $\sigma=P(\varphi)$ and let $g:[0, \infty) \rightarrow X$ be the smallest $\varphi$-gradient ray starting at $e$. Assume $n \geq 0$.

(1) $\gamma_{n}(\sigma)=g(n)$.

(2) $V_{n}(\sigma)=N(g([n, n+L]), R)$.

(3) $f_{n}(\sigma)$ is the restriction to $V_{n}(\sigma)$ of the primitive $h$ of $\varphi$ with $h(e)=0$.

Definition 2.25. Let $\sigma \in \Sigma$. We say that $\sigma$ is consistent if for all $i, j \geq 0$, we have

$$
f_{i}(\sigma)(x)=f_{j}(\sigma)(x)
$$

for all $x \in V_{i}(\sigma) \cap V_{j}(\sigma)$. We denote by $\Sigma(\infty)$ the set of all consistent sequences.

Lemma 2.26 [Coornaert and Papadopoulos 2001, Lemma 6.8]. $P\left(\Phi_{0}\right) \subseteq \Sigma(\infty)$.

Theorem 2.27 [Coornaert and Papadopoulos 2001, Theorem 7.18]. The set of consistent sequences $\Sigma(\infty)$ is a shift of finite type. Moreover $\left(\Phi_{0}, \alpha\right)$ and $(\Sigma(\infty), T)$ are conjugate via the map $P$.

\section{The topological entropy of $\Sigma(\infty)$}

Let $\Gamma$ be a Gromov hyperbolic group with a finite generating set $A$ on which we fix a total order relation. Let $\Sigma(\infty)$ the corresponding SFT.

For $n \in \mathbb{N}$, we denote by $W_{n}$ the set of all words with length $n$ that occur in $\Sigma(\infty)$ and by $A_{n}$ the set of all elements in $\Gamma$ with word length $n$ with respect to the finite generating set $A$ (as a particular case, $A_{0}=\{e\}$ ). We set $D_{n}=\bigcup_{1 \leq k \leq n} W_{k}$ and $B_{n}=\bigcup_{0 \leq k \leq n} A_{k}$. For each $s \in S$, we set

$$
W_{n}(s)=\left\{\left(\sigma_{0}, \ldots, \sigma_{n-1}\right) \in W_{n} \mid \sigma_{0}=s\right\},
$$

and for each $a \in A$,

$$
A_{n}(a)=\left\{a \gamma \in A_{n} \mid \gamma \in A_{n-1}\right\} .
$$

We write $D_{n}(s)=\bigcup_{1 \leq k \leq n} W_{k}(s)$ and $B_{n}(a)=\bigcup_{1 \leq k \leq n} A_{n}(a)$.

We denote by $\operatorname{gr}(\Gamma, A)$ the growth entropy of $\Gamma$ with respect to $A$ :

$$
\operatorname{gr}(\Gamma, A)=\lim _{n \rightarrow \infty} \frac{1}{n} \log \operatorname{card} A_{n} .
$$


We also define

$$
\begin{aligned}
\bar{A}_{n} & =\left\{\gamma \in A_{n} \mid \gamma=w\left(\sigma_{0}\right) \cdots w\left(\sigma_{n-1}\right) \text { for some }\left(\sigma_{0}, \ldots, \sigma_{n-1}\right) \in W_{n}\right\}, \\
\bar{B}_{n} & =\bigcup_{1 \leq k \leq n} \bar{A}_{n}, \\
\bar{A}_{n}(w(s)) & =\left\{\gamma \in A_{n}(w(s)) \mid \gamma=w\left(\sigma_{0}\right) \cdots w\left(\sigma_{n-1}\right)\right. \\
\bar{B}_{n}(w(s)) & =\bigcup_{1 \leq k \leq n} \bar{A}_{n}(w(s)) .
\end{aligned}
$$

Lemma 3.1. There is a constant $K>0$ such that

$$
\operatorname{card}\left\{\left(\sigma_{0}, \ldots, \sigma_{n-1}\right) \in W_{n} \mid w\left(\sigma_{0}\right) \ldots w\left(\sigma_{n-1}\right)=\gamma\right\} \leq K,
$$

for every $n \geq 1$ and every $\gamma \in A_{n}$.

Proof. Let $\varphi, \varphi^{\prime} \in \Phi_{0}$ and $g, g^{\prime}$ their smallest gradient rays starting at $e$ such that $g(n)=g^{\prime}(n)=\gamma \in A_{n}$. Note that $\varphi(\gamma, e)=\varphi^{\prime}(\gamma, e)=-n$. We denote $\sigma=P(\varphi)$ and $\sigma^{\prime}=P\left(\varphi^{\prime}\right)$. By Lemma 2.24, we have $\gamma_{n}(\sigma)=\gamma_{n}\left(\sigma^{\prime}\right)=\gamma$.

We first claim that $g=g^{\prime}$ on $[0, n]$. We now assume that $g \neq g^{\prime}$ on $[0, n]$. We may assume that $g^{\prime}<g$ in the lexicographic order on $A^{\mathbb{N}}$ without loss of generality.

Note that $\varphi(e, \gamma)=\varphi(g(0), g(n))=n=|e-\gamma|$, and $g^{\prime}:[0, n] \rightarrow X$ is a geodesic joining $e$ and $\gamma$. From Proposition 2.9(2) it follows that $g^{\prime}:[0, n] \rightarrow X$ is a $\varphi$-gradient arc. Then we define the path $\bar{g}:[0, \infty) \rightarrow X$ by

$$
\bar{g}(k)= \begin{cases}g^{\prime}(k) & \text { for } 0 \leq k \leq n, \\ g(k) & \text { for } n \leq k\end{cases}
$$

By Lemma 2.8, the path $\bar{g}$ is $\varphi$-gradient ray starting at $e$ such that $\bar{g}<g$ in the lexicographic order on $A^{\mathbb{N}}$. Therefore $g$ would be not the smallest $\varphi$-gradient ray. Hence we have $g=g^{\prime}$ on $[0, n]$.

Let $h, h^{\prime}$ be primitives for $\varphi, \varphi^{\prime}$ satisfying $h(e)=h^{\prime}(e)=0$, respectively. We set $B=B(\gamma, L+R)$.

We secondly claim that if $h=h^{\prime}$ on $B$, then $h=h^{\prime}$ on $N(g([0, n+L]), R)$. Notice that $R>16 \delta$ and $L>2 R$. Let $k \in[0, n]$ satisfying $n-k \leq 2 R$. Since $N(g([k, n+L]), R) \subseteq B$, we have $h=h^{\prime}$ on $N(g([k, n+L]), R)$. Next let $k \geq 0$ satisfying $n-k>2 R$. For $x \in B(g(k), R)$, we have

$$
\begin{aligned}
n & =|g(0)-g(n)|=|g(0)-g(k)|+|g(k)-g(n)| \\
& \geq|g(0)-x|-|x-g(k)|+|g(k)-g(n)| \geq|g(0)-x|-R+(n-k) \\
& >|g(0)-x|+R>|g(0)-x|+16 \delta .
\end{aligned}
$$

By Proposition 2.14, we have $\varphi(x, g(n))=\operatorname{dist}\left(x, R_{\varphi, n}\right)$. Recall that

$$
R_{\varphi, n}=\{x \in X \mid \varphi(x, g(n))=0\} \cap B(g(n), 16 \delta) .
$$


Hence $h(x)+n=\operatorname{dist}\left(x, R_{\varphi, n}\right)$. This shows that the value of $h(x)$ depends only on the restriction of $h$ on $B(g(n), 16 \delta) \subseteq B$. Namely we obtain our claim.

We now assume that $h=h^{\prime}$ on $B$. In this case, we remark that $g=g^{\prime}$ on $[0, n+L]$. By Proposition 2.21, we have $V\left(\alpha^{k}(\varphi)\right)=N\left(g(k)^{-1} g([k, k+L]), R\right)=V\left(\alpha^{k}\left(\varphi^{\prime}\right)\right)$ for $0 \leq k \leq n$. For each $x \in V\left(\alpha^{k}(\varphi)\right)$, since

$$
N\left(g(k)^{-1} g([k, k+L]), R\right)=g(k)^{-1} N(g([k, k+L]), R),
$$

there is $y \in N(g([k, k+L]), R)$ such that $x=g(k)^{-1} y$. Then

$$
\rho\left(\alpha^{k}(\varphi)\right)(x)=g(k)^{-1} \varphi(x, e)=\varphi(y, g(k))=h(y)-h(g(k))=h(y)+k .
$$

Similarly we also obtain $\rho\left(\alpha^{k}(\varphi)\right)(x)=h^{\prime}(y)+k$. Hence if $h=h^{\prime}$ on $B$, then it follows from the second claim that

$$
\rho\left(\alpha^{k}(\varphi)\right)(x)=h(y)+k=h^{\prime}(y)+k=\rho\left(\alpha^{k}\left(\varphi^{\prime}\right)\right) .
$$

Therefore $\rho\left(\alpha^{k}(\varphi)\right)=\rho\left(\alpha^{k}\left(\varphi^{\prime}\right)\right)$; that is, $\sigma_{k}=\sigma_{k}^{\prime}$ for all $0 \leq k \leq n$.

Hence it suffices to set $K=(2(L+R)+1)^{b}$, where $b=\operatorname{card} B=\operatorname{card} B(e, L+R)$. Indeed, for every $x \in B$ we have, using Proposition 2.6,

$$
|h(x)+n|=|h(x)-h(\gamma)|=|\varphi(x, \gamma)| \leq|x-\gamma| \leq L+R .
$$

This easily leads to the assertion.

Corollary 3.2. $h_{\text {top }}(\Sigma(\infty)) \leq \operatorname{gr}(\Gamma, A)$.

Proof. For each $n \geq 0$, the map $W_{n} \ni\left(\sigma_{0}, \ldots, \sigma_{n-1}\right) \mapsto w\left(\sigma_{0}\right) \cdots w\left(\sigma_{n-1}\right) \in A_{n}$ is uniformly finite-to-one by Lemma 3.1. Thus

$$
\text { card } W_{n} \leq K \operatorname{card} A_{n} .
$$

The assertion follows immediately.

Remark 3.3. A fundamental theorem of J. Stallings [1971] shows that a finitely generated group $\Gamma$ has infinitely many ends if and only if it has a form of either (2) or (3) of Corollary 1.2. In particular, a torsion-free group has the form (1).

\section{Proof of main results}

Proof of Corollary 1.2. In view of Corollary 3.2, we just need to show that $h_{\text {top }}(\Sigma(\infty)) \geq \operatorname{gr}(\Gamma, A)$ if one of the conditions (1)-(3) of Corollary 1.2 is satisfied. Remark 3.3 shows that it suffices to check cases (2) and (3); but we check case (1) explicitly as well because it is very simple.

Case (1): It suffices to show that the map $\left(\sigma_{0}, \ldots, \sigma_{n-1}\right) \mapsto w\left(\sigma_{0}\right) \cdots w\left(\sigma_{n-1}\right)$ from $W_{n}$ to $A_{n}$ is surjective. Let $\gamma \in A_{n}$. There is the smallest geodesic segment $r:[0, n] \rightarrow X$ from $e$ to $\gamma$. We can take $g$ to be a geodesic ray extending $r$, meaning 
that $r(k)=g(k)$ for all $0 \leq k \leq n$. Indeed, by assumption, we have $\Gamma=G_{1} * G_{2}$. Then $\gamma$ is written as a reduced word $g_{1} \cdots g_{m}$, where $g_{k} \in G_{i_{k}}$ with $i_{k} \neq i_{k+1}$ for $1 \leq k \leq m-1$. Hence for $l \geq 1$, it is enough to set

$$
g(n+2 l)=\gamma \cdot \underbrace{a b \cdots a b}_{2 l}, \quad g(n+2 l-1)=\gamma \cdot \underbrace{a b \cdots b a}_{2 l-1},
$$

for some $a \in F_{i}$ and $b \in F_{i_{m}}$ with $i \neq i_{m}$ and $a, b \neq e$.

We consider the cocycle $\varphi_{g}$ having the Busemann function $h_{g}$ as a primitive. It is clear that $g$ is a $\varphi_{g}$-gradient ray. Moreover by definition, $g$ is, in fact, the smallest $\varphi_{g}$-gradient ray starting at $e$. Hence $\gamma=w\left(P\left(\varphi_{g}\right)\right) \cdots w\left(P\left(\alpha^{n-1}\left(\varphi_{g}\right)\right)\right)$. It follows that the map above is surjective. Thus card $A_{n} \leq \operatorname{card} W_{n}$, and $\operatorname{gr}(\Gamma, A) \leq$ $h_{\text {top }}(\Sigma(\infty))$ as needed.

Case (2): Now we assume that $\Gamma=G_{1} *_{H} G_{2}$. Let $\gamma \in A_{n}$ with $n \geq 2$. We express the element $\gamma$ by the reduced word $g_{1} \cdots g_{m}$, where $g_{k} \in G_{i_{k}} \backslash H$ with $i_{k} \neq i_{k+1}$ for $1 \leq k \leq m-1$. We take a sequence $\left(g_{m+1}, g_{m+2}, \ldots\right)$ such that $g_{k} \in F_{i_{k}} \backslash H$ with $i_{k-1} \neq i_{k}$ for all $k \geq m+1$. We define a sequence $(g(k))_{k=1}^{\infty}$ in $X$ by $g(k)=g_{1} \ldots g_{k}$ for $k \geq 1$. Let $\langle y, z\rangle=\frac{1}{2}(|y|+|z|-|y-z|)$ be the Gromov product based at $e$. For $l \geq k \geq m$,

$$
\begin{aligned}
2\langle g(k), g(l)\rangle & =|g(k)|+|g(l)|-\left|g(k)^{-1} g(l)\right| \\
& \geq k+l-\left|g(k)^{-1} g(l)\right|=k+l-\left|g_{k+1} \cdots g_{l}\right| \\
& \geq k+l-(l-k)=2 k
\end{aligned}
$$

tends to $\infty$ with $k$; thus there exists $\xi \in \partial X$ such that the sequence $(g(k))_{k=1}^{\infty}$ converges to $\xi$. Let $r:[0, \infty) \rightarrow X$ be a geodesic ray starting at $e$ with $r(\infty)=$ $\xi$. We denote by $\varphi_{r}$ the cocycle with respect to the Busemann function $h_{r}$. Let $g^{\prime}:[0, \infty) \rightarrow X$ be the smallest $\varphi_{r}$-gradient ray starting at $e$. Because $r$ is also a $\varphi_{r}$-gradient ray, it follows from Proposition 2.11 that $g^{\prime}(\infty)=\xi$. We can express $g^{\prime}$ by the infinite reduced word $\left(g_{1}^{\prime}, g_{2}^{\prime}, \ldots\right)$ with $g_{k}^{\prime} \in G_{j_{k}} \backslash H$ and $j_{k} \neq j_{k+1}$ for $k \geq 1$. Since $g^{\prime}(\infty)=\xi$, we have $i_{k}=j_{k}$ for all $k \geq 1$. Moreover we obtain $\gamma=g(m)=g_{1} \cdots g_{m}=g_{1}^{\prime} \cdots g_{m}^{\prime} h$ for some $h \in H$. Let $k_{m} \geq 1$ such that $g^{\prime}\left(k_{m}\right)=$ $g_{1}^{\prime} \cdots g_{m}^{\prime}$. Then we have $\left|g(m)-g^{\prime}\left(k_{m}\right)\right| \leq 1$. Note that $n-1 \leq k_{m} \leq n+1$. Hence we have proved that for any $\gamma \in A_{n}$, there is $\gamma^{\prime} \in A_{n}$ such that $\gamma^{\prime} \in B(\gamma, 2)$ and $\gamma^{\prime}=w\left(\sigma_{0}\right) \cdots w\left(\sigma_{n-1}\right)$ for some $\left(\sigma_{0}, \ldots, \sigma_{n-1}\right) \in W_{n}$. Therefore card $A_{n} \leq$ card $B(e, 2) \cdot$ card $W_{n}$, and the assertion follows.

Case (3): We assume that $\Gamma=G *_{H} \theta$. Let $\gamma \in A_{n}$. The element $\gamma$ can be

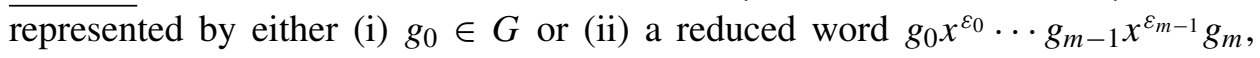
where $g_{k} \in G$ and $\varepsilon_{k} \in\{1,-1\}$ for all $0 \leq k \leq m$. In case (i), we set $g_{k}=e$ for $k \geq 1$ and $\varepsilon_{k}=1$ for $k \geq 0$. In case (ii), we set $g_{k}=e$ for $k \geq m+1$ and $\varepsilon_{k}=\varepsilon_{m-1}$ 
for $k \geq m$. Then we define the sequence $(g(k))_{k=0}^{\infty}$ in $X$ by $g(k)=g_{0} x^{\varepsilon_{0}} \cdots g_{k} x^{\varepsilon_{k}}$ for all $k \geq 0$. Again, for $l \geq k \geq m$,

$$
2\langle g(k), g(l)\rangle=|g(k)|+|g(l)|-\left|g(k)^{-1} g(l)\right| \geq k+l-\left|x^{\varepsilon_{k+1}} \cdots x^{\varepsilon_{l}}\right|=2 k
$$

goes to $\infty$ with $k$; hence $(g(k))_{k=0}^{\infty}$ converges to some $\xi \in \partial \Gamma$. Let $r:[0, \infty) \rightarrow X$ be a geodesic ray with $r(0)=e$ and $r(\infty)=\xi$. We denote by $\varphi_{r}$ the cocycle with respect to the Busemann function $h_{r}$. Let $g^{\prime}:[0, \infty) \rightarrow X$ be the smallest $\varphi_{r^{-}}$ gradient ray starting at $e$. We can also represent the geodesic ray $g^{\prime}$ as the infinite reduced word $\left(g_{0}^{\prime} x^{\delta_{0}}, g_{1}^{\prime} x^{\delta_{1}}, \ldots\right)$. Since $g^{\prime}(\infty)=\xi$, we have $\varepsilon_{i}=\delta_{i}$ for all $i \geq 0$. Moreover we obtain $\gamma=g_{0} x^{\varepsilon_{0}} \cdots g_{m}=g_{0}^{\prime} x^{\varepsilon_{0}} \cdots g_{m}^{\prime} g$, for some either $g \in H$ if $\varepsilon_{m}=1$, or $g \in \theta(H)$ if $\varepsilon_{m}=-1$. Let $k_{m} \geq 1$ such that $g^{\prime}\left(k_{m}\right)=g_{0}^{\prime} x^{\varepsilon_{0}} \cdots g_{m}^{\prime}$. Then we have $\left|\gamma-g\left(k_{m}\right)\right| \leq 1$. Note that $n-1 \leq k_{m} \leq n+1$. Hence we have shown that for each $\gamma \in A_{n}$, there is $\gamma^{\prime} \in A_{n}$ such that $\gamma^{\prime} \in B(\gamma, 2)$ and $\gamma^{\prime}=w\left(\sigma_{0}\right) \cdots w\left(\sigma_{n-1}\right)$ for some $\left(\sigma_{0}, \ldots, \sigma_{n-1}\right) \in W_{n}$. Therefore card $A_{n} \leq \operatorname{card} B(e, 2) \cdot \operatorname{card} W_{n}$, and $h_{\text {top }}(\Sigma(\infty)) \leq \operatorname{gr}(\Gamma, A)$ as needed.

Remark 4.1. It is easy to check that the topological entropy $h_{\text {top }}(\Sigma(\infty))$ does not depend on the choice of total order relations on $A$.

Proof of Theorem 1.1. It suffices to show that $h_{\text {top }}(\Sigma(\infty)) \leq k_{\infty}^{-}\left(\lambda_{A}\right)$, because the inequality $k_{\infty}^{-}\left(\lambda_{A}\right) \leq \operatorname{gr}(\Gamma, A)$ has been proved in [Okayasu 2004, Proposition 4.1]. Let $\lambda_{w(S)}=\left\{\lambda_{w(s)} \mid s \in S\right\}$. Note that $k_{\infty}^{-}\left(\lambda_{w(S)}\right) \leq k_{\infty}^{-}\left(\lambda_{A}\right)$.

Since $\Sigma(\infty)$ is an SFT, there are $N \in \mathbb{N}$ and $W \subseteq S^{N+1}$ such that

$$
\Sigma(\infty)=\left\{\left(\sigma_{n}\right)_{n \geq 0} \in \Sigma \mid\left(\sigma_{n}, \ldots, \sigma_{n+N}\right) \in W \text { for any } n \geq 0\right\} .
$$

Let $I=S^{N}$ and $\beta_{N}: \Sigma(\infty) \rightarrow I^{\mathbb{N}}$ be the $N$-th higher block code. Then the subshift $\beta_{N}(\Sigma(\infty))$ is the Markov shift $\Sigma_{M}$ for some matrix $M=[M(i, j)]_{i, j \in I}$. Let $\mu$ be the maximal measure on $\Sigma(\infty)$, i.e., $h_{\text {top }}(\Sigma(\infty))=h_{\mu}\left(\left.T\right|_{\Sigma(\infty)}\right)$. For simplicity, we denote by $h$ the topological entropy of $\Sigma(\infty)$. We denote by $\left[\sigma_{0}, \ldots, \sigma_{n-1}\right]$ the cylinder set at 0 -th coordinate. For $\left(\sigma_{0}, \ldots, \sigma_{n-1}\right) \in W_{n}$ with $n \geq N$, we have

$$
\mu\left(\left[\sigma_{0}, \ldots, \sigma_{n-1}\right]\right)=\frac{l_{i} r_{j}}{e^{(n-N) h}},
$$

where $i=\left(\sigma_{0}, \ldots, \sigma_{N-1}\right), j=\left(\sigma_{n-N}, \ldots, \sigma_{n-1}\right) \in I$ and $l, r$ are the left and right Perron vectors of $M$ with $\sum_{i \in I} l_{i} r_{i}=1$ (see [Kitchens 1998]).

For each $n \geq 0$, denote by $P_{n}$ the projection onto the subspace

$$
\overline{\operatorname{span}}\left\{\delta_{\gamma} \in \ell^{2}(\Gamma)|| \gamma \mid=n\right\} .
$$

For $a \in A$, define the partial isometry $T_{a} \in \mathbb{B}\left(\ell^{2}(\Gamma)\right)$ [Okayasu 2002; 2004] by

$$
T_{a}=\sum_{n \geq 0} P_{n+1} \lambda_{a} P_{n}
$$


For each $s \in S$, we define $X_{s}$ by

$$
\sum_{n \geq 1} \sum_{\substack{\left(\sigma_{0}, \sigma_{1}, \ldots, \sigma_{n-1}\right) \\ \in W_{n}(s)}} \mu\left(\left[\sigma_{0}, \sigma_{1}, \ldots \sigma_{n-1}\right]\right) T_{w\left(\sigma_{1}\right)} \cdots T_{w\left(\sigma_{n-1}\right)} P_{0} T_{w\left(\sigma_{n-1}\right)}^{*} \cdots T_{w\left(\sigma_{1}\right)}^{*} T_{w\left(\sigma_{0}\right)}^{*} .
$$

Then $\sum_{s \in S}\left[X_{s}, \lambda_{w(s)}\right]=P_{0}$, because

$\sum_{s \in S} \lambda_{w(s)} X_{s}$

$$
\begin{aligned}
& =\sum_{n \geq 1} \sum_{s \in S} \sum_{\substack{\left(\sigma_{0}, \ldots, \sigma_{n-1}\right) \\
\in W_{n}(s)}} \mu\left(\left[\sigma_{0}, \ldots \sigma_{n-1}\right]\right) T_{w\left(\sigma_{0}\right)} \cdots T_{w\left(\sigma_{n-1}\right)} P_{0} T_{w\left(\sigma_{n-1}\right)}^{*} \cdots T_{w\left(\sigma_{0}\right)}^{*} \\
& =\sum_{n \geq 1} \sum_{\substack{\left(\sigma_{0}, \ldots, \sigma_{n-1}\right) \\
\in W_{n}}} \mu\left(\left[\sigma_{0}, \ldots \sigma_{n-1}\right]\right) T_{w\left(\sigma_{0}\right)} \cdots T_{w\left(\sigma_{n-1}\right)} P_{0} T_{w\left(\sigma_{n-1}\right)}^{*} \cdots T_{w\left(\sigma_{0}\right)}^{*}
\end{aligned}
$$

and

$$
\begin{aligned}
& \sum_{s \in S} X_{S} \lambda_{w(s)} \\
& =\sum_{n \geq 1} \sum_{s \in S} \sum_{\substack{\left.\sigma_{0}, \ldots, \sigma_{n-1}\right) \\
\in W_{n}(s)}} \mu\left(\left[\sigma_{0}, \sigma_{1}, \ldots \sigma_{n-1}\right]\right) T_{w\left(\sigma_{1}\right)} \cdots T_{w\left(\sigma_{n-1}\right)} P_{0} T_{w\left(\sigma_{n-1}\right)}^{*} \cdots T_{w\left(\sigma_{1}\right)}^{*} \\
& =P_{0}+\sum_{n \geq 1} \sum_{\substack{\left(\sigma_{0}, \ldots, \sigma_{n-1}\right) \\
\in W_{n}}} \mu\left(\left[\sigma_{0}, \sigma_{1}, \ldots \sigma_{n-1}\right]\right) T_{w\left(\sigma_{0}\right)} \cdots T_{w\left(\sigma_{n-1}\right)} P_{0} T_{w\left(\sigma_{n-1}\right)}^{*} \cdots T_{w\left(\sigma_{0}\right)}^{*} .
\end{aligned}
$$

Next we give an estimate of $\left\|X_{s}\right\|_{1}^{\tilde{+}}$. For $n \in \mathbb{N}$ and $\gamma \in \bar{A}_{n}(w(s))$, we define

$$
s_{\gamma}=\sum_{\substack{\left(\sigma_{0}, \ldots, \sigma_{n-1}\right) \in W_{n}(s) \\ \gamma=w\left(\sigma_{0}\right) \cdots w\left(\sigma_{n-1}\right)}} \mu\left(\left[\sigma_{0}, \ldots, \sigma_{n-1}\right]\right) .
$$

This sum is uniformly finite by Lemma 3.1. Thus

$$
C_{1} e^{-n h} \leq s_{\gamma} \leq C_{2} e^{-n h}
$$

for constants $C_{1}, C_{2}>0$, independent of $n$ and $\gamma$.

Let $s_{1} \geq s_{2} \geq \cdots$ be the eigenvalues of $\left(X_{s}^{*} X_{s}\right)^{1 / 2}$. For each $j \in \mathbb{N}$, there is $\gamma_{j} \in \bar{A}_{n_{j}}(w(s))$ such that $s_{j}=s_{\gamma_{j}}$.

Let $\varepsilon>0$. Recall that

$$
\left\|X_{s}\right\|_{1}^{\widetilde{+}}=\inf _{Y \in \mathbb{F}\left(\ell^{2}(\Gamma)\right)_{1}^{+}}\left\|X_{s}-Y\right\|_{1}^{+} .
$$

By doing finite rank perturbations if necessary, we may assume that for all $j \geq 1$,

$$
e^{-n_{j}(h+\varepsilon)} \leq s_{j} \leq e^{-n_{j}(h-\varepsilon)} .
$$


Let $N \in \mathbb{N}$ with $e^{-N \varepsilon} \leq C_{1}$ and $n \geq N$. If there is $m>n$ such that $j \leq$ card $\bar{B}_{n}(w(s))$ and $\gamma_{j} \in \bar{A}_{m}(w(s))$, we have

$$
e^{-m(h-\varepsilon)} \geq e^{-n(h+\varepsilon)} .
$$

For otherwise we would have

$$
s_{j} \leq e^{m(h-\varepsilon)}<e^{-n(h+\varepsilon)} \leq e^{-n \varepsilon} \frac{s_{\gamma}}{C_{1}} \leq s_{\gamma}
$$

for all $\gamma \in \bar{B}_{n}(w(s))$ and this is a contradiction. Therefore $e^{m(h-\varepsilon)} \geq e^{-n(h+\varepsilon)}$, namely

$$
m \leq n \frac{h+\varepsilon}{h-\varepsilon} .
$$

We put

$$
k=\max \left\{m \in \mathbb{N} \mid m \leq n \frac{h+\varepsilon}{h-\varepsilon}\right\} .
$$

Since

$$
\mu([s])=\sum_{\left(\sigma_{0}, \ldots, \sigma_{n-1}\right) \in W_{n}(s)} \mu\left(\left[\sigma_{0}, \ldots, \sigma_{n-1}\right]\right) \leq \operatorname{card} W_{n}(s) \cdot C e^{-n h},
$$

for some $C>0$, we obtain

$$
\frac{\mu([s]) e^{n h}}{C} \leq \operatorname{card} W_{n}(s) .
$$

Hence

$$
\begin{aligned}
\left\|X_{s}\right\|_{1}^{\tilde{+}} & \leq \limsup _{n \rightarrow \infty} \frac{\sum_{j=1}^{\operatorname{card} \bar{B}_{n}(w(s))} s_{j}}{\sum_{j=1}^{\operatorname{card} \bar{B}_{n}(w(s))} j^{-1}} \\
& \leq \limsup _{n \rightarrow \infty} \frac{\sum_{l=1}^{k} \sum_{\gamma \in \bar{A}_{l}(w(s))} \sum_{w\left(\sigma_{0}\right) \cdots w\left(\sigma_{l-1}\right)=\gamma} \mu\left(\left[\sigma_{0}, \ldots, \sigma_{l-1}\right]\right)}{\log \operatorname{card} \bar{B}_{n}(w(s))} \\
& =\limsup _{n \rightarrow \infty} \frac{\sum_{l=1}^{k} \mu([s])}{\log \operatorname{card} \bar{B}_{n}(w(s))} \\
& \leq \limsup _{n \rightarrow \infty} \frac{n}{\log \operatorname{card} \bar{A}_{n}(w(s))} \frac{h+\varepsilon}{h-\varepsilon} \mu([s]) \\
& \leq \limsup _{n \rightarrow \infty} \frac{n}{\log \operatorname{card} W_{n}(s)-\log K} \frac{h+\varepsilon}{h-\varepsilon} \mu([s]) \\
& \leq \limsup _{n \rightarrow \infty} \frac{h \log \mu([s])+n h-\log C-\log K}{h-\varepsilon} \mu([s]) \\
& =\frac{h+\varepsilon}{h(h-\varepsilon)} \mu([s]) .
\end{aligned}
$$


Here we have used that card $W_{n}(s) \leq K \operatorname{card} \bar{A}_{n}(w(s))$ (Lemma 3.1). Since $\varepsilon>0$ is arbitrary, we have

$$
\left\|X_{s}\right\|_{1}^{\widetilde{+}} \leq \frac{1}{h} \mu([s]) .
$$

Thanks to Proposition 2.1, we obtain

$$
h=h_{\text {top }}(\Sigma(\infty)) \leq k_{\infty}^{-}\left(\lambda_{w(S)}\right) \leq k_{\infty}^{-}\left(\lambda_{A}\right) \leq \operatorname{gr}(\Gamma, A) .
$$

\section{Acknowledgment}

The author expresses his gratitude to Masaki Izumi for his constant encouragement and important suggestions.

\section{References}

[Coornaert and Papadopoulos 1993] M. Coornaert and A. Papadopoulos, Symbolic dynamics and hyperbolic groups, Lecture Notes in Mathematics 1539, Springer, Berlin, 1993. MR 94d:58054 Zbl 0783.58017

[Coornaert and Papadopoulos 2001] M. Coornaert and A. Papadopoulos, "Horofunctions and symbolic dynamics on Gromov hyperbolic groups", Glasg. Math. J. 43:3 (2001), 425-456. MR 2003c: 20047 Zbl 1044.20027

[David and Voiculescu 1990] G. David and D. Voiculescu, "s-numbers of singular integrals for the invariance of absolutely continuous spectra in fractional dimensions", J. Funct. Anal. 94:1 (1990), 14-26. MR 92f:47014 Zbl 0732.47023

[Gohberg and Krĕ̌n 1969] I. C. Gohberg and M. G. Kreĭn, Introduction to the theory of linear nonselfadjoint operators, Translations of Mathematical Monographs 18, American Mathematical Society, Providence, R.I., 1969. MR 39 \#7447 Zbl 0181.13504

[Gromov 1987] M. Gromov, "Hyperbolic groups", pp. 75-263 in Essays in group theory, edited by S. M. Gersten, Math. Sciences Research Inst. Publ. 8, Springer, New York, 1987. MR 89e:20070 Zbl 0634.20015

[Kitchens 1998] B. P. Kitchens, Symbolic dynamics: One-sided, two-sided and countable state Markov shifts, Universitext, Springer, Berlin, 1998. MR 98k:58079 Zbl 0892.58020

[Lind and Marcus 1995] D. Lind and B. Marcus, An introduction to symbolic dynamics and coding, Cambridge University Press, Cambridge, 1995. MR 97a:58050 Zbl 00822672

[Matsumoto 1997] K. Matsumoto, "On $C^{*}$-algebras associated with subshifts", Internat. J. Math. 8:3 (1997), 357-374. MR 98h:46077 Zbl 0885.46048

[Okayasu 2002] R. Okayasu, "Cuntz-Krieger-Pimsner algebras associated with amalgamated free product groups”, Publ. Res. Inst. Math. Sci. 38 (2002), 147-190. MR 2003h:46102 Zbl 1007.46055

[Okayasu 2004] R. Okayasu, "Entropy of subshifts and the Macaev norm", J. Math. Soc. Japan 56:1 (2004), 177-191. MR 2004j:47137 Zbl 1062.47030

[Stallings 1971] J. Stallings, Group theory and three-dimensional manifolds, Yale Mathematical Monographs 4, Yale University Press, New Haven, 1971. MR 54 \#3705 Zbl 0241.57001

[Voiculescu 1979] D. Voiculescu, "Some results on norm-ideal perturbations of Hilbert space operators”, J. Operator Theory 2:1 (1979), 3-37. MR 80m:47012 Zbl 0446.47003

[Voiculescu 1981] D. Voiculescu, "Some results on norm-ideal perturbations of Hilbert space operators. II”, J. Operator Theory 5:1 (1981), 77-100. MR 83f:47014 Zbl 0483.46036 
[Voiculescu 1990] D. Voiculescu, "On the existence of quasicentral approximate units relative to normed ideals. I", J. Funct. Anal. 91:1 (1990), 1-36. MR 91m:46089 Zbl 0762.46051

[Voiculescu 1993] D. Voiculescu, "Entropy of random walks on groups and the Macaev norm", Proc. Amer. Math. Soc. 119:3 (1993), 971-977. MR 93m:47077 Zbl 0799.60006

Received September 23, 2003. Revised May 7, 2005.

\section{RUI OKAYASU}

DEPARTMENT OF MATHEMATICS

OSAKA KYOIKU UNIVERSITY

ASAHIGAOKA KASHIWARA 582-8582

JAPAN

rui@cc.osaka-kyoiku.ac.jp 